\title{
Age-related changes of protein SUMOylation balance in the A $\beta$ PP Tg2576 mouse model of Alzheimer's disease
}

\section{Robert Nisticò ${ }^{1,2}$, Caterina Ferraina ${ }^{3}$, Veronica Marconi ${ }^{2}$, Fabio Blandini ${ }^{4}$, Lucia Negri ${ }^{2}$, Jan Egebjerg ${ }^{5}$ and Marco Feligioni ${ }^{3}$ *}

1 IRCCS Fondazione Santa Lucia, Rome, Italy

2 Department of Physiology and Pharmacology, Sapienza University of Rome, Rome, Italy

${ }^{3}$ Laboratory of Pharmacology of Synaptic Plasticity, EBRI "Rita Levi-Montalcini" Foundation, Rome, Italy

${ }^{4}$ Center for Research in Neurodegenerative Diseases, C. Mondino National Neurological Institute, Pavia, Italy

${ }^{5}$ Neuroscience Drug Discovery DK, H. Lundbeck A/S, Valby, Denmark

\section{Edited by:}

Cesare Mancuso, Catholic

University School of Medicine, Italy

Reviewed by:

Isidro Ferrer, University of

Barcelona, Spain

Zuner Assis Bortolotto, University of

Bristol, UK

\section{*Correspondence:}

Marco Feligioni, Laboratory of

Synaptic Plasticity, EBRI "Rita

Levi-Montalcini" Foundation, Via del

Fosso di Fiorano 64/65,

00143 Rome, Italy

e-mail:m.feligioni@ebri.it
Alzheimer's disease (AD) is a complex disorder that affects the central nervous system causing a severe neurodegeneration. This pathology affects an increasing number of people worldwide due to the overall aging of the human population. In recent years SUMO protein modification has emerged as a possible cellular mechanism involved in AD. Some of the proteins engaged in the physiopathological process of AD, like BACE1, GSK3- $\beta$ tau, $A \beta P P$, and JNK, are in fact subject to protein SUMO modifications or interactions. Here, we have investigated the SUMO/deSUMOylation balance and SUMO-related proteins during the onset and progression of the pathology in the Tg2576 mouse model of AD. We examined four age-stages $(1.5,3,6,17$ months old) and observed shows an increase in SUMO-1 protein conjugation at 3 and 6 months in transgenic mice with respect to WT in both cortex and hippocampus. Interestingly this is paralleled by increased expression levels of Ubc9 and SENP1 in both brain regions. At 6 months of age also the SUMO-1 mRNA resulted augmented. SUMO-2-ylation was surprisingly decreased in old transgenic mice and was unaltered in the other time windows. The fact that alterations in SUMO/deSUMOylation equilibrium occur from the early phases of AD suggests that global posttranslational modifications may play an important role in the mechanisms underlying disease pathogenesis, thus providing potential targets for pharmacological interventions.

Keywords: sumoylation, Tg2576, Alzheimer's disease, SENP1, Ubc9, SUMO-1, SUMO-2/3, neurodegeneration

\section{INTRODUCTION}

Alzheimer's disease $(\mathrm{AD})$ is considered one of the most common and debilitating pathologies in the elderly. AD is a slowly progressive neurodegenerative disease that is characterized by impairment of memory and eventually by other symptoms (Heun et al., 2013).

Research indicates that the disease is associated with the production of oligomers of amyloid beta $(A \beta)$ leading to progressive neuritic plaque deposition and hyperphosphorylation of microtubule protein tau with subsequent formation of neurofibrillary tangles (Tiraboschi et al., 2004).

An "oxidative stress hypothesis" for $\mathrm{AD}$ has been recently postulated (Markesbery, 1997; Di Domenico et al., 2011; Leitao et al., 2011), albeit it is still unclear whether oxidative stress represents a trigger mechanism to unbalance normal cell functions or might be rather the consequence of pathogenic events. SUMOylation is among the PTMs that has been recently linked to $\mathrm{AD}$ (Lee et al., 2013). In fact, SUMOylation induces critical changes on $\mathrm{AD}$-associated proteins like microtubule-associated protein tau (MAPT), amyloid $\beta$ precursor protein (A $\beta \mathrm{PP}$ ) (Georgopoulou et al., 2001; Marcus and Schachter, 2011), c-Jun terminal kinase (JNK) (Feligioni et al., 2011; Sclip et al., 2013) and AMPA receptors (Jaafari et al., 2013), that play an important role in neuronal physiology (Pittaluga et al., 2005, 2006; Holman et al., 2007), therefore providing novel targets for therapeutic intervention. SUMO family includes at least three paralogs (SUMO-1 to -3) ubiquitously expressed in all organism tissues including the brain (Droescher et al., 2013). SUMOs target proteins through non-covalent or covalent interactions affecting their cellular localization, aggregation, metabolism and activity (Steffan et al., 2004; Martin et al., 2007; Feligioni et al., 2011; Krumova et al., 2011; Droescher et al., 2013). Protein SUMOylation has recently been recognized to play a fundamental role in oxidative stress (Bossis and Melchior, 2006; Feligioni et al., 2011; Leitao et al., 2011; Feligioni and Nisticò, 2013), in the regulation of glutamate release (Feligioni et al., 2009) and also in the modification of activity of several intracellular proteins like A $P$ PP and tau (Dorval and Fraser, 2006, 2007; Zhang and Sarge, 2008). As a consequence, SUMO-mediated alterations in specific intracellular signaling pathways could promote AD pathogenesis. Altogether, these observations indicate that SUMOylation could play an important role in the onset of $\mathrm{AD}$, though its precise contribution still remains elusive. Here we performed an age-related analysis on the expression levels of global protein 
SUMOylation and SUMO-related enzymes in the Tg2576 mouse modeling $\mathrm{AD}$. Importantly, we observe significant differences in SUMO/deSUMOylation balance at an early stage of the pathology.

\section{MATERIALS AND METHODS ETHICS STATEMENT}

All experiments were done in accordance with the European Community Council Directive of 24 November 1986 (86/609/EEC) and approved by the Ethical Committee on animal experiments of EBRI "Rita Levi-Montalcini” Foundation (Rome, Italy).

\section{BRAIN TISSUE DISSECTION}

Adult male and female Tg2576 (Brecht et al., 2005) mice were sacrificed by cervical dislocation and immediately after hippocampal and cortical brain regions were dissected on ice. Both Tg2576 and WT mice were processed in parallel and were used for the experiments at the different age stages $(1.5,3,6$, and 17 months). Once removed, brain tissues were immediately placed in a cryopreservation solution \{containing $0.32 \mathrm{M}$ sucrose, buffered to $\mathrm{pH}$ 7.4 with Tris-(hydroxymethyl)-amino methane [Tris, final concentration (f.c.) $0.01 \mathrm{M}$ ] $\}$ and stored at $-80^{\circ} \mathrm{C}$ until needed. The cortical and hippocampal tissues of $\operatorname{Tg} 2576$ and their wild-type (WT) littermates have been kindly provided by Lundbeck AS (Denmark).

\section{PREPARATION OF LYSATE FROM BRAIN TISSUE}

Around $200 \mu \mathrm{g}$ of mice tissues were lysed in $300 \mu \mathrm{l}$ of Lysis Buffer solution (LB) made up of $1 \%$ Triton X-100 (Serva, Germany), complete protease inhibitor cocktail solution (Serva, Germany), phosphatase inhibitor cocktail solution (Serva, Germany), $20 \mathrm{mM}$ of NEM (Sigma-Aldrich) and the following components (mM): TRIS acetate, 20; sucrose, 0.27; EDTA, 1; EGTA, 1; Na Orthovanadate, 1; NaF, 50; Na Pyrophosphate, 5; Na $\beta$-glycerophosphate, 10; DTT, 1.

Samples were then kept for $30 \mathrm{~min}$ on ice to allow protein solubilization. Later a centrifugation step of $10 \mathrm{~min}$ at $12000 \mathrm{rpm}$ was applied to the samples and the supernatant was collected and stored at $-20^{\circ} \mathrm{C}$ until needed.

\section{WESTERN BLOT}

Protein concentrations for each sample were determined by Bradford assay and the samples were directly analyzed by immunoblotting following resuspension in Laemmli buffer.

Equal amount of proteins ( $\sim 15 \mu \mathrm{g}$ for each condition) were resolved by $10 \%$ SDS-polyacrylamide gels and blotted onto PVDF membrane (Serva, Germany). The proteins blotted on the membrane were then blocked for $1 \mathrm{~h}$ at room temperature using Tris-buffered saline-Tween (t-TBS) (M) Tris, 0, 02; NaCl, 0, 15; Tween 20, 0, 1\%) containing 5\% skimmed milk.

Later the membranes were treated with specific antibodies and the incubation last for $12 \mathrm{~h}$ at $4^{\circ} \mathrm{C}$ with mild agitation. The primary antibody used for western blot analysis are: rabbit amyloid precursor protein (A $\beta P P$ ) 1:2000 (SigmaAldrich, USA), rabbit SUMO-1 1:1000 (Cell Signaling, USA), rabbit SUMO-2/3 (18H8) 1:1000 (Cell Signaling, USA), rabbit
SENP1 1:500 (Thermo scientific, USA), mouse UBC9 (C12) 1:1000 (Santa Cruz Biotechnology, USA), mouse $\beta$-actin 1:30000 (Sigma-Aldrich, Italy).

Tris-buffer saline solution (TBS) with $0,1 \%$ of Tween 20 was used to wash the membranes for 50 min of wash in t-TBS. Then the blots were incubated for $1 \mathrm{~h}$ at room temperature with peroxidase-conjugated goat anti-rabbit or anti-mouse IgG secondary antibodies (UCS Diagnostic), as needed.

After $50 \mathrm{~min}$ of washes in t-TBS bands immunoreactivity was detected by enhanced chemiluminescence (ECL; WESTAR, Cyanagen, Italy). For all experiments stripping procedure was applied when the control of loading, performed blotting for $\beta$-actin, was required (stripping buffer from SignaGen, USA).

For each time point, a western blot has been performed in which proteins from six samples for WT and Tg2576 have been separated and analyzed. Where possible, membranes have been stripped and re-blotted for different antibodies with the purpose of using same loading conditions.

\section{RNA EXTRACTION AND REAL-TIME PCR}

Total RNA was extracted from brain cortex and hippocampus using the Trizol reagent (Invitrogen, Carlsbad, CA) according to the manufacturer's instruction. RNA yield and purity were determined by spectrophotometry absorption at 260 and $280 \mathrm{~nm}$. To obtain cDNA, an equal amount of mRNA ( $1 \mu \mathrm{g})$ underwent to Reverse Transcription (Promega, Madison, WI). The resulting cDNA was stored at $-20^{\circ} \mathrm{C}$ until used for the further analysis. Messenger RNA expression was quantitatively measured with quantitative (q) real time PCR using iCycler Bio-Rad. The reaction was performed in a $25 \mu$ l volume using SensiMix SYBR Green and Fluorescein kit (Bioline, London, UK). All the measures were performed in triplicate. The reaction conditions were as follows: $95^{\circ} \mathrm{C}$ for $10 \mathrm{~min}$ (Polymerase activation), followed by 40 cycles at $95^{\circ} \mathrm{C}$ for $15,55-50^{\circ} \mathrm{C}$ (Temp. depends on the $\mathrm{Tm}$ of primers) for $15 \mathrm{~s}$ and $72^{\circ} \mathrm{C}$ for $15 \mathrm{~s}$. The reaction mixture without the cDNA was used as control.

The primer sequences used in this study were as following for SUMO-1: forward 5'-GCCTGGGACATGGGTTT-3' and reverse $5^{\prime}$-TTAATGAAGCTGGTACAGACGATG-3'; SUMO2: forward 5'-GGCAGGGTTTGTCAATGAGGC-3' and reverse 5'-CTGGAGTAAAGTA GTAGCAGGCTC-3'; SUMO-3: forward $5^{\prime}$-GAGGCAGGGCTTGTCAATGAG-3' and reverse $5^{\prime}$-GGTC AGGACAACGGTTGGGTG-3'; SENP1: forward 5'-AATGGCTG ATGATGATGTG- $3^{\prime}$ and reverse $5^{\prime}$-TTGGACAAGGATTAGA CTGAAT-3'; UBC9: forward 5'-CATCCAGCCTTCGT AAA CC- $3^{\prime}$ and reverse $5^{\prime}$-GCTAACAGGCAGGGAGAT- ${ }^{\prime}$; glyceraldehydes-3-phosphate dehydrogenase (GAPDH): forward $5^{\prime}$-GCCA AGGCTGTGGGCAAGGT-3' and reverse $5^{\prime}$-TCTCCAGGCGGC ACGTCAGA-3'.

The $C t$ values of the specific gene of interest were normalized to the $C t$ value of the endogenous control, GAPDH, and the comparative $C t$ method $\left(2^{-\Delta \Delta C t}\right)$ was then applied using WT mice group as calibrator.

\section{STATISTICAL ANALYSIS}

In the western blots and real time PCR experiments, $T$-test analysis was performed and $p<0.05$ was considered statistically significant. Statistical analysis for biochemical experiments 
was performed using GraphPad PRISM 5. A number (as indicated in figure legends) of animal tissues has been used at the same time point for the experiments. Values shown represent the mean \pm s.e.m. Final western blots have been run using representative sampling

\section{RESULTS}

\section{A $\beta$ PP INCREASES IN Tg2576 MICE}

The Tg2576 transgenic mouse carries a transgene coding for the 695 -amino acid isoform of human A $\beta$ PP derived from a large Swedish family with early-onset AD (Hsiao et al., 1996). This mouse model of $\mathrm{AD}$ expresses high concentrations of mutant $\mathrm{A} \beta$, develops a significant number of amyloid plaques, and displays functional deficits like decreased dendritic spine density, impaired long-term potentiation (LTP), and behavioral deficits (Jacobsen et al., 2006; Balducci et al., 2011).

In order to validate the mice model used in this work we measured A $\beta P P$ immunoreactivity in the cortical and hippocampal tissues from the brains of Tg2576 and WT mice. Tg2576 are expected to have an augmented expression of A $\beta P P$ because, beside the murine endogenous $\mathrm{A} \beta \mathrm{PP}$, they genetically over-express human mutated $\mathrm{A} \beta \mathrm{PP}$. Indeed, both cortex and hippocampus prepared from Tg2576 mice showed a more intense immunoreactivity of the $\mathrm{A} \beta \mathrm{PP}$ band corresponding to 95-100 kDa compared to WT (Figure 1D).

\section{PROTEIN SUMOylation CHANGES IN THE CORTEX OF Tg2576 MICE DURING ONTOGENESIS}

The cerebral cortex is among the most vulnerable brain regions affected by the disease showing progressive plaque burden and tissue atrophy (Tosun et al., 2011). Although in a less severe manner, some biochemical hallmarks have also been reported in different AD mice models (Sclip et al., 2011; Izco et al., 2014).

In order to evaluate protein SUMOylation changes during disease progression, we dissected and lysated cortical tissues from 1.5, 3, 6, 17 months old Tg2576 and their age-matched WT littermates. Protein SUMOylation has been revealed by western blotting using an anti-SUMO- 1 and an anti-SUMO-2/3 antibody. SUMO- 1 and SUMO2/3 for each time point has been normalized against the actin. Interestingly, the ratio in protein SUMO-1ylation between Tg2576 and WT mice is increased at early stages, and it later decreases following a bell-shaped curve. In fact, a peak of SUMO-1-ylation is observed at 3 and 6 months old animals (Figure 1A, 3 months: $1.65 \pm 0.16 p<0.05$; 6 months: $1.78 \pm 0.08 p<0.01)$.

Noteworthy a protein SUMOylated band, absent in the other ages analyzed, appears at 6 months of age. The immunoreactivity of this band results more intense in the Tg2576 mice compared to controls (Figure 1A, black arrow). Further studies are required to identify which protein becomes target of SUMO-1 at this stage, and whether this protein modification has a role in $\mathrm{AD}$ pathology.

SUMO-2/3-ylation did not show any differences in the ratio between Tg2576 and WT mice at different ages except for 17 months old mice, where it resulted drastically decreased in transgenic mice (Figure 1B, 17 months: $0.35 \pm 0.05 p<0.01$ ). Further experiments are required to understand what implication this reduction may have. As for the SUMO-1 western blot, also for SUMO-2/3 a more intense band is detectable (Figure 1B, black arrow).

\section{PROTEIN SUMOylation CHANGES IN THE HIPPOCAMPUS OF Tg2576 MICE DURING ONTOGENESIS}

The hippocampus is the part of the brain that is involved in memory formation, being one of the first regions to suffer damage in AD. Since Tg2576 show cognitive impairment caused by hippocampal dysfunction even before the onset of the pathology (D'Amelio et al., 2011), we analyzed protein SUMOylation changes between Tg2576 and WT mice also in this brain area.

Similarly to the cortex, SUMO-1-ylation is increased in the hippocampus of 3 and 6 months old Tg2576 compared to WT (Figure 2A, 3 months: $1.71 \pm 0.07 p<0.01$; 6 months: $2.07 \pm 0.39 p<0.01)$.

SUMO-2/3-ylation did not show any differences in the ratio between Tg2576 and WT mice at different ages except for 17 months old, where surprisingly it resulted decreased in transgenic mice (Figure 2B, 17 months: $0.65 \pm 0.01 p<0.01$ ). This result needs more investigation in order to understand its potential implication in the pathophysiology of AD.

\section{SUMO PATHWAY RELATED PROTEINS IN Tg2576 TRANSGENIC MICE ONTOGENESIS}

The protein conjugation by SUMO occurs through the activity of four key enzymes which form the SUMOylation pathway. Among them Ubc9 and SENP1 are the most important since they are dedicated exclusively to protein SUMOylation. On the one hand Ubc9 binds and transports SUMO to the target protein, on the other SENP1 is the isopeptidase that regulates the deSUMOylation (Droescher et al., 2013; Feligioni and Nisticò, 2013). Ubc9 and SENP1 are critical for the maintenance of SUMO/deSUMOylation balance, therefore their expression levels have been assessed by western blotting in cortical and hippocampal tissue from Tg2576 and WT mice. The expression level of the two proteins has been normalized on actin and the ratio between Tg2576 and WT has been analyzed.

Interestingly, we found that the expression level of Ubc9 is modulated both in cortical and hippocampal tissue. In fact, the western blots show for both WT and Tg2576 an increase of Ubc9 from 3 to 17 months with a peak at 6 months of age. Moreover Ubc9 seems to be highly expressed in Tg2576 compared to WT at different ages (Figure 1C, 6 months: $1.57 \pm 0.16$ $p<0.05$ ) (Figure 2C, 3 months: $1.42 \pm 0.24 p<0.05$; 6 months: $2.22 \pm 0.37 p<0.01)$. Conversely, immunoreactivity of SENP1 seems to be augmented in the cortex of transgenic mice at 3 and 6 months and only at 6 months in hippocampus of Tg2576 compared to age-matched WT animals (Figure 1C, 3 months: $1.75 \pm 0.21 p<0.05$; 6 months: $2.51 \pm 0.21 p<0.01$ ) (Figure 2C, 6 months: $2.15 \pm 0.42 p<0.01$ ).

\section{SUM0-1-, SUM0-2-, SUM0-3-, SENP1- AND Ubc9-mRNA IN BRAIN CORTEX AND HIPPOCAMPUS}

SUMO-1 mRNA levels in the cortex (Figure 3A) and hippocampus (Figure 4A) of Tg2576 mice at 1.5 months of age were similar to the levels measured in WT mice, whereas at 6 months of age their levels were significantly higher than that of WT mice. 

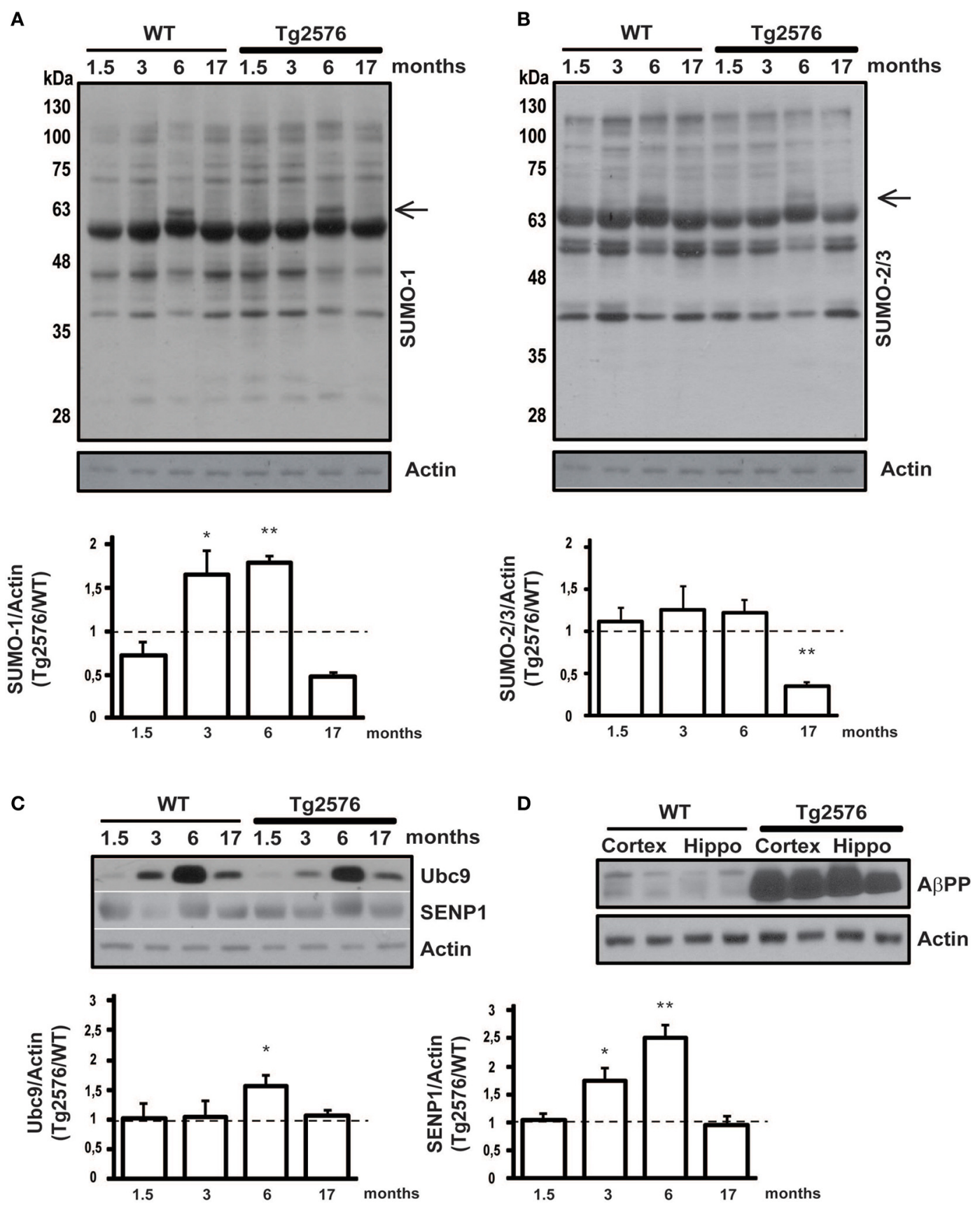

FIGURE 1 | Protein SUMOylation and SUMO-related proteins expression level in cortical tissue. Representative western blots of WT and Tg2576 mice cortical tissue lysate. Cortex of WT and Tg2576 animals was analyzed at four time points $(1.5,3,6$, and 17 months of age). Anti-SUMO-1 (A), anti-SUMO-2/3 (B), anti-Ubc9,
anti-SENP1 (C), and anti-AßPP (D) have been used for the protein detections. Results are expressed as ratio between Tg2576 and WT. Data represent means \pm s.e.m. of 6 animals for each age. (t-test) ${ }^{* *} p<0.01$ or ${ }^{*} p<0.05$ Tg2576 vs. WT. $\beta$-Actin was used as loading control.
On the contrary, we did not find any significant changes in SUMO-2, SUMO-3, SENP1, and Ubc9 mRNA levels in the cortex (Figures 3B-E) and hippocampus (Figures 4B-E) of Tg2576 and WT mice at the age of 1.5 and 6 months. The SUMO-1 mRNA increase is in accordance with the elevated level of SUMO1-ylation that has been found in cortex and hippocampus at 6 months of age. It seems that SUMO-1 synthesis is augmented in order to be disposable for conjugation, whereas the Ubc9 and SENP1 protein levels are sufficient in both tissues to maintain SUMOylation pathway functional. The SUMO-2 and -3 mRNA level is in line with protein SUMO-2/3-ylation expression that resulted unchanged up to 6 months of age. 

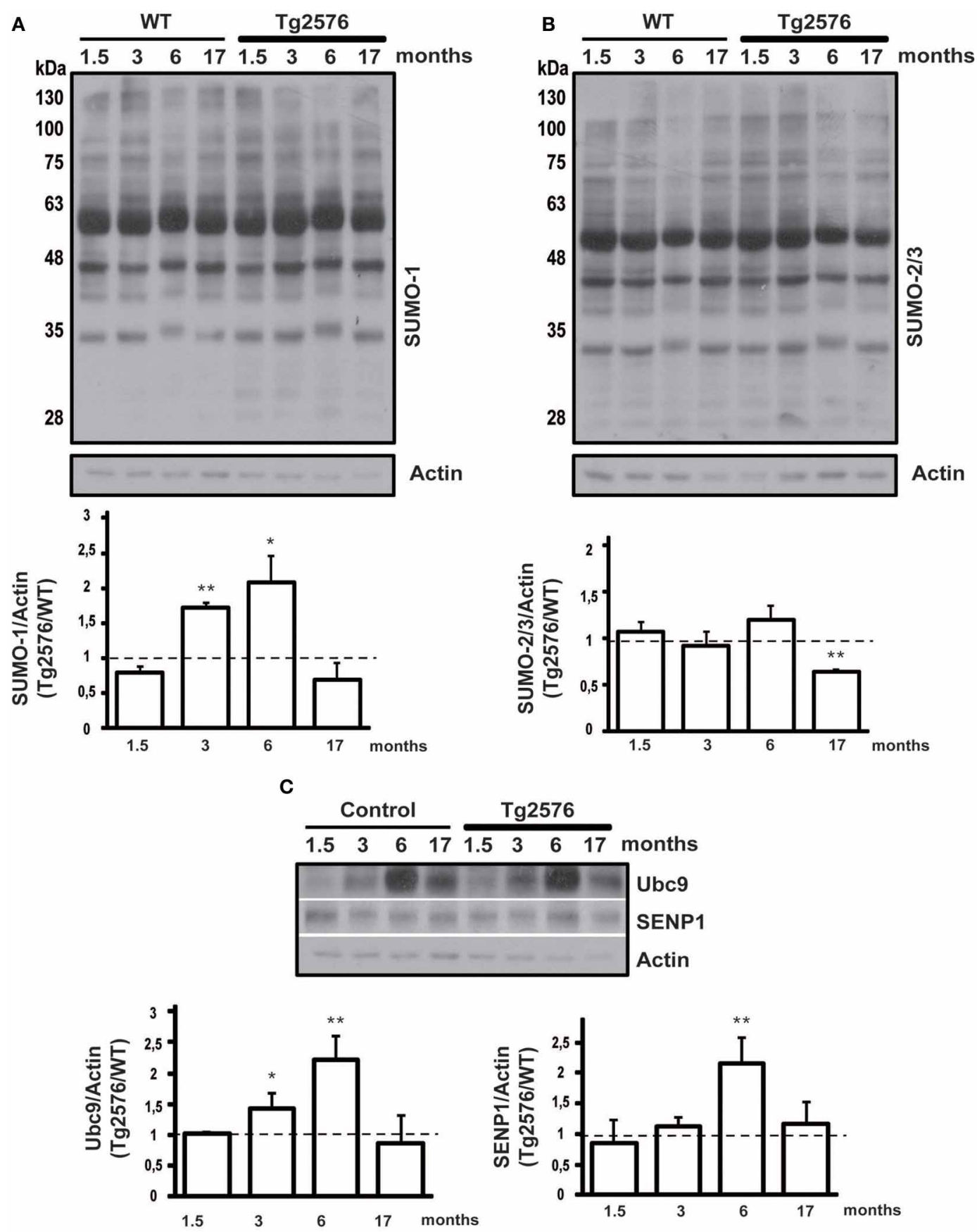

FIGURE 2 | Protein SUMOylation and SUMO-related proteins expression level in hippocampal tissue. Representative western blots of WT and Tg2576 mice hippocampal tissue lysate.

Hippocampus of WT at four time points $(1.5,3,6$, and 17 months of age) was compared with their age-matched Tg2576 mice.

\section{DISCUSSION}

Tg2576 mice model were developed to test the amyloid hypothesis of AD. These mice model answered partially the question whether $\mathrm{AD}$ pathology comes from the accumulation of $\mathrm{A} \beta$ species. $\mathrm{A} \beta$ species, although poorly detectable, can be found in the soluble form (monomers, dimers, trimmers or oligomers) already at a
Anti-SUMO-1 (A), anti-SUMO-2/3 (B), anti-Ubc9 and anti-SENP1 (C) have been used for protein detections. Results are expressed as ratio between Tg2576 and WT. Data represent means \pm s.e.m. of 6 animals for each age. (t-test) ${ }^{* *} p<0.01$ or ${ }^{*} p<0.05$ Tg2576 vs. WT. $\beta$-Actin was used as loading control. young age ( $<5$ months old) (Kawarabayashi et al., 2001; Klingner et al., 2003; Lesné et al., 2006), but they reach a highly detectable level around 6-8 months (Hsiao et al., 1996). Insoluble amyloid plaques start to form at around 9-10 months in hippocampus and cortex (Hsiao et al., 1996). This model also shows the typical $\mathrm{AD}$ cognitive impairment with a mild onset at 3 months, which 

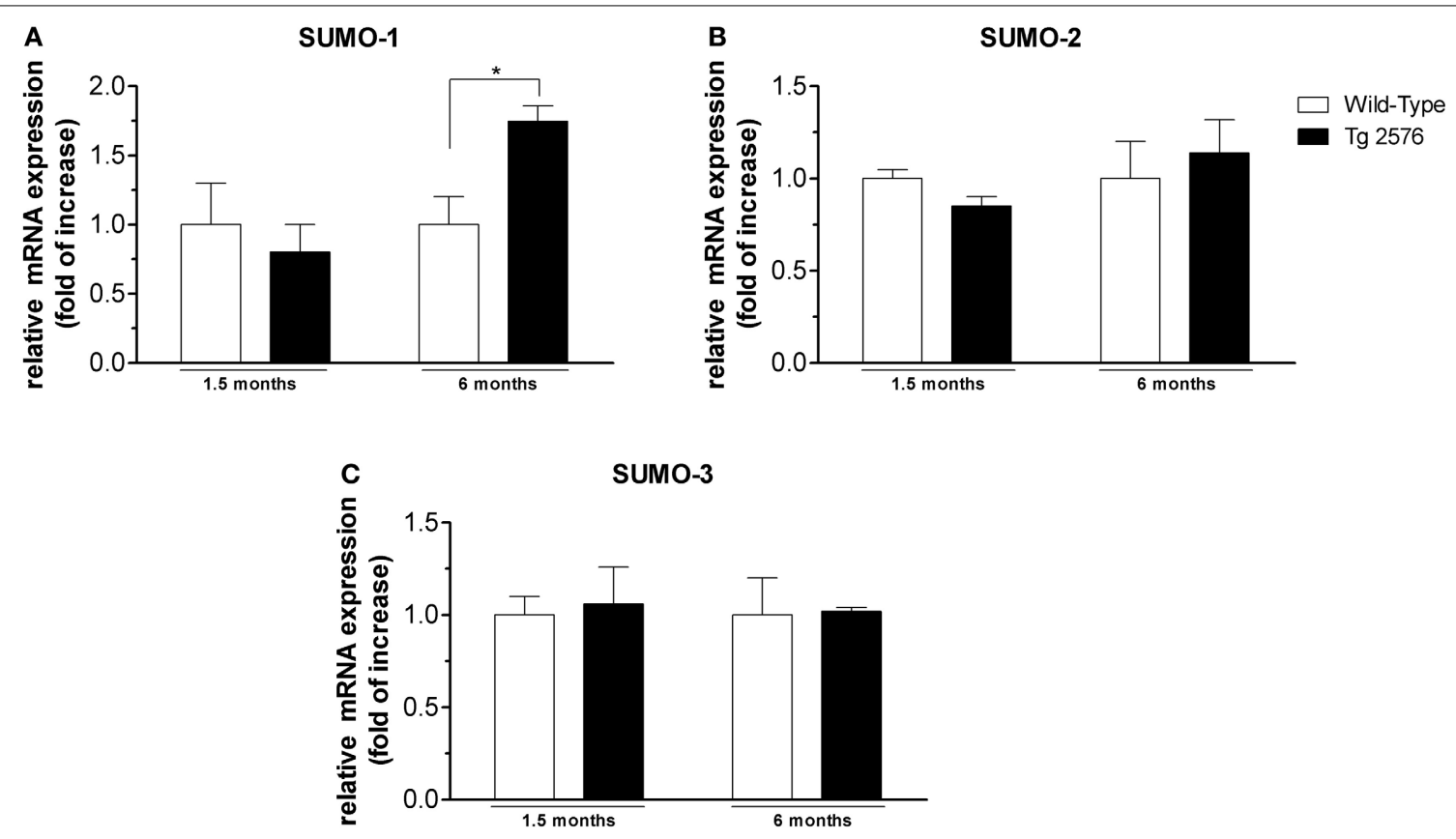

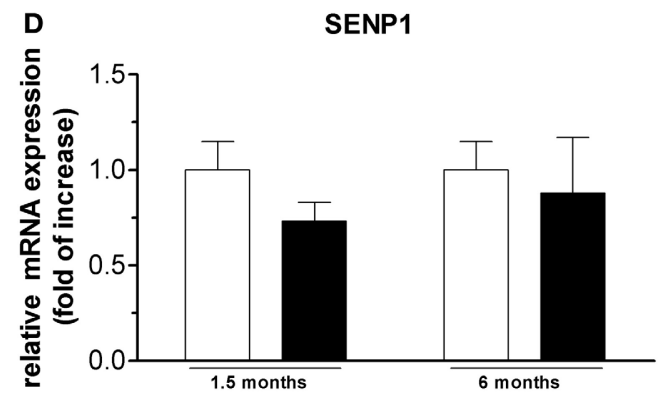

FIGURE 3 | mRNA expression level of SUMO and SUMO-related proteins in cortical tissues. Analyses of SUMO-1 (A), SUMO-2 (B), SUMO-3 (C), SENP1 (D), Ubc9 (E) mRNA expression in brain cortex of Tg2576 and WT mice at the age of 1.5 and 6 months. The mRNA expression levels,

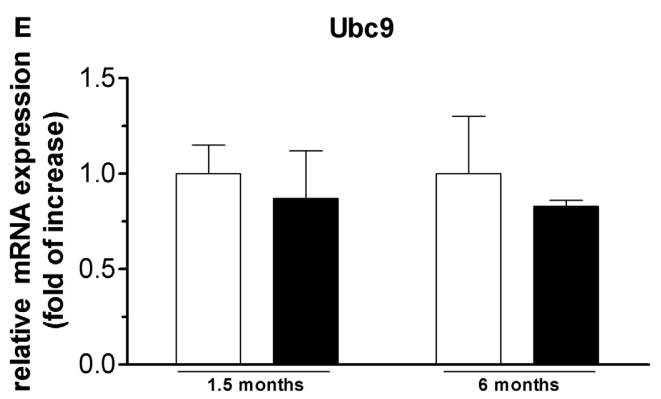

determined by real-time polymerase chain reaction (RT-PCR), were expressed in relation to glyceraldehyde 3-phosphate dehydrogenase (GAPDH) and presented as fold increase relative to WT animals. Data represent mean \pm s.e.m. of 4 animals. ( $t$-test) ${ }^{*} p<0.05$ Tg2576 vs. WT. is exacerbated starting from 6 months of age. However, Tg2576 do not resemble all the features of $\mathrm{AD}$ since they do not display any formation of neurofibrillary tangles, marked neuronal loss or gross brain atrophy, (Irizarry et al., 1997), but are anyway a very useful model to study AD amyloid-related pathology (Deacon et al., 2008).

Several AD-associated proteins undergo SUMO protein modification, leading to hypothesize that SUMO plays a fundamental role in $\mathrm{AD}$ pathogenesis. Despite that, we still lack information about how SUMO/deSUMOylation process is altered during $\mathrm{AD}$ onset and development. Therefore a better understanding of SUMO changes during $\mathrm{AD}$ pathogenesis could help to disclose the role of protein modification in the onset of AD. Notably, McMillan and co-authors (McMillan et al., 2011) reported that no clear differences in global protein SUMOylation can be measured in adult $\operatorname{Tg} 2576$ (9 months old) vs. their respective $\mathrm{WT}$.

In this manuscript, we decided to investigate SUMOylation changes during mice lifespan analyzing four time points.
To our knowledge this is the first ontogenetic study of SUMO/deSUMOylation profile in an AD model.

Yun and co-authors have shown in in-vitro experiments that SUMO- 1 can be considered a modulator of the synthesis of A $\beta$ oligomers (Yun et al., 2013). In fact SUMO-1, but not SUMO-2 or -3 , when overexpressed in neuronal or cell culture, increases BACE1 level which mediates the amiloydogenic cleavage of A $\beta P P$. Moreover, as previously reported in 18 months old A $\beta P P$ transgenic mice (Yun et al., 2013) SUMO-1 free protein is increased in their cortical tissue. So, it has been postulated that the increase of $\mathrm{A} \beta$ accumulation together with an accrual of cell oxidative stress can act synergistically to enhance protein SUMO-1-ylation. By conjugating BACE1, SUMO-1 intensifies A $\beta$ oligomers production (Yun et al., 2013). Our data show that already at the age of 3 months, protein SUMO-1-ylation is augmented both in cortical and hippocampal tissue of Tg2576 model compared to their respective WT.

Therefore it can be hypothesized that at early stages (3 months) the "cell stress" might contribute more to the increase 

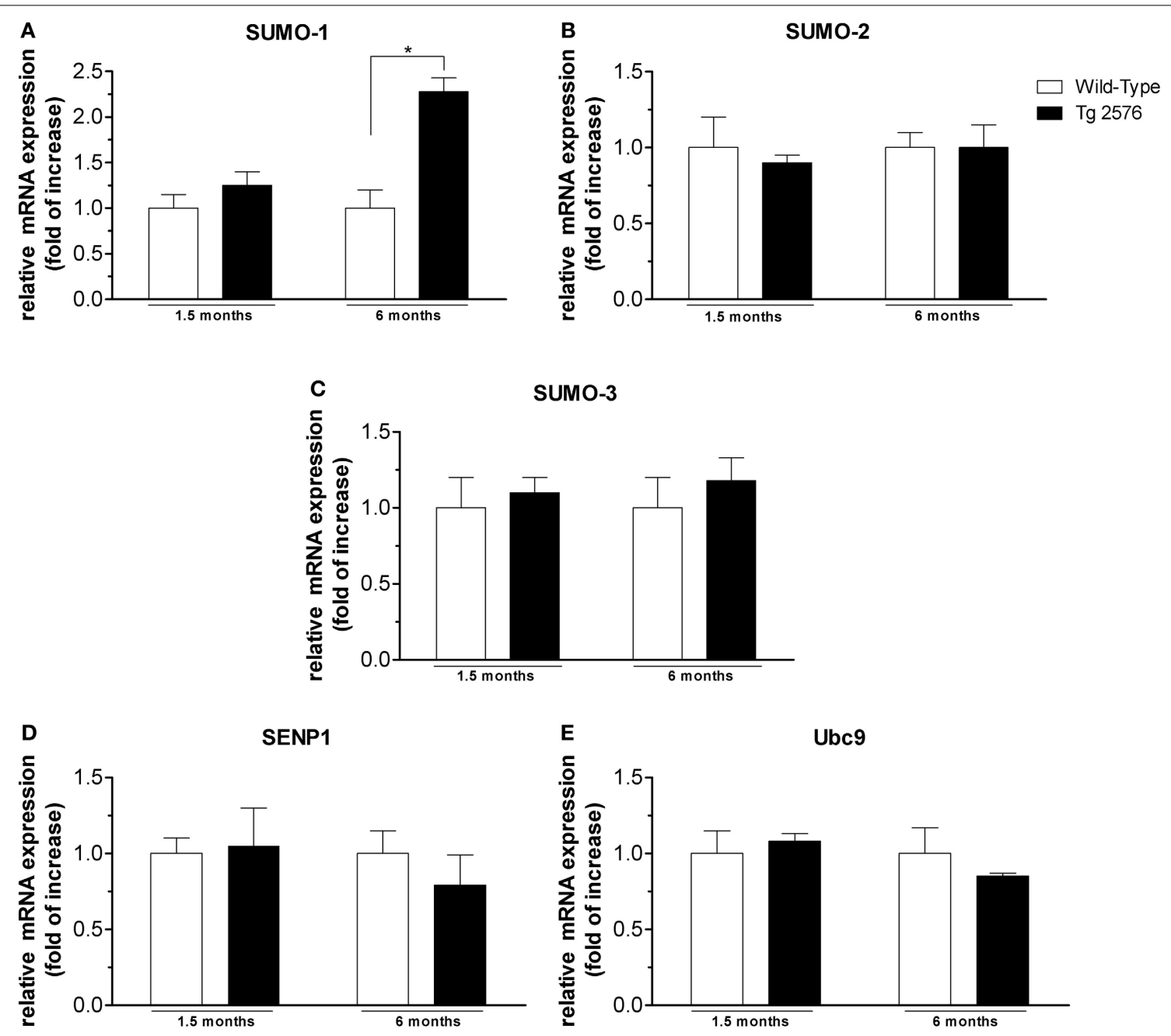

FIGURE 4 | mRNA expression level of SUMO and SUMO-related proteins in hippocampal tissues. Analyses of SUMO-1 (A), SUMO-2 (B), SUMO-3 (C), SENP1 (D), Ubc9 (E) mRNA expression in brain hippocampus of Tg2576 and WT mice at the age of 1.5 and 6 months. The mRNA expression levels,

determined by real-time polymerase chain reaction (RT-PCR), were expressed in relation to glyceraldehyde 3-phosphate dehydrogenase (GAPDH) and presented as fold increase relative to WT animals. Data represent mean \pm s.e.m. of 4 animals. ( $t$-test) $* p<0.05$ Tg2576 vs. WT. in SUMO-1-ylation compared to $\mathrm{A} \beta$ oligomers which are present at low levels at this age. As a result, SUMO-1-ylation activates multiple signaling pathways, like BACE1, GSK3 $\beta$, JNK activation, that can contribute to boost the production of $A \beta$ oligomers. The feedback loop established between SUMO-1, A $\beta$ oligomers and cell stress cooperate to the development of AD neuropathology. Interestingly, SUMO-2-ylation does not change between Tg2576 and WT except for 17 months old transgenic mice where it is drastically diminished both in cortex and hippocampus. This aspect also deserves a deeper investigation since very little is known about SUMO-2/3 contribution to AD.

Ubc9 and SENP1 are critical enzymes for protein SUMOylation (Droescher et al., 2013; Feligioni and Nisticò, 2013). An increase of SUMOylation is associated with the onset of several pathologies including cancer. For example, Ubc9 expression, the E2 enzyme which facilitates SUMOylation, has been reported increased in primary colon and prostate cancer compared with normal tissue (Moschos et al., 2010). We here demonstrate that the increase of SUMOylation in Tg2576 brain tissues corresponds to a high expression level of Ubc9 at the same time points. Therefore Ubc9 plays a fundamental role for SUMOylation event also in $\mathrm{AD}$. On the other hand, the over-functionality of SUMOylation in Tg2576 is also supported by an elevated presence of the SUMO protease SENP1. Our results are in line with previous data where SENP-1 was found increased during oxygen-glucose deprivation (OGD) experiments, suggesting that the neuronal response could involve a complex interplay between SUMOylation and deSUMOylation (Cimarosti et al., 2012).

The analysis of mRNA expression has shown an increase of SUMO-1 RNA only at the age of 6 months both in cortex and hippocampus. This is in line with our western blot data where the major increase of SUMOylation was exactly found at 6 months of age in $\mathrm{Tg} 2576$ mice, where probably there is a need for more protein synthesis. In contrast, although we reported an increase in protein expression level of both Ubc9 and SENP1, the mRNA 
expression of both proteins was unchanged. We can therefore speculate that the degradation system of Ubc9 and SENP1 could be decreased during lifespan of mice. Further studies should be carried out in order to better clarify this aspect. In line with a previous work (McMillan et al., 2011), our experiments did not show significant changes in protein SUMO-2/3-ylation in AD mice up to 6 months of age. SUMO-2/3 role in AD remains still elusive. However, in in-vitro experiments it has been shown that SUMO2 increases $\mathrm{N}$-terminal ADAM-cleaved A $\beta$ PP fragment ( $\alpha$-NTF) while a mutant form of SUMO-2 lacking the SUMOylation activity secreted significantly more $\mathrm{N}$-terminal BACE1-cleaved A $\mathrm{PP}$ fragment ( $\beta$-NTF) and A $\beta$ (Li et al., 2003). In line with this report, we can speculate that the reduction in protein SUMO-2/3-ylation that was observed in 17 months old Tg2576 mice is concomitant with an elevated amyloid deposition which occurs at this stage in $\mathrm{AD}$ mice model (Jacobsen et al., 2006). In conclusion, here we report for the first time that protein SUMO/deSUMOylation equilibrium is unbalanced in a mouse model of $\mathrm{AD}$ at a very early stage of the pathology. We believe that this event can contribute to the onset of $\mathrm{AD}$ pathogenesis. Future investigation on the target(s) of protein SUMOylation at an early stage could possibly lead to the identification of novel pharmacological targets.

\section{ACKNOWLEDGMENTS}

We thank Miss Marisa Hipwood for excellent language revision and Mr. Guglielmo Barberini for technical support. This work was partly supported by grants from: "H. Lundbeck Foundation, Denmark" and from the Italian Ministry of Health to IRCCS Neurological Institute "C. Mondino" (Ricerca Corrente 20132015).

\section{REFERENCES}

Balducci, C., Mehdawy, B., Mare, L., Giuliani, A., Lorenzini, L., Sivilia, S., et al. (2011). The $\gamma$-secretase modulator CHF5074 restores memory and hippocampal synaptic plasticity in plaque-free Tg2576 mice. J. Alzheimer's Dis. 24, 799-816. doi: 10.3233/JAD-2011-101839

Bossis, G., and Melchior, F. (2006). Regulation of SUMOylation by reversible oxidation of SUMO conjugating enzymes. Mol. Cell 21, 349-357. doi: 10.1016/j.molcel.2005.12.019

Brecht, S., Kirchhof, R., Chromik, A., Willesen, M., Nicolaus, T., Raivich, G., et al. (2005). Specific pathophysiological functions of JNK isoforms in the brain. Eur. J. Neurosci. 21, 363-377. doi: 10.1111/j.1460-9568.2005.03857.x

Cimarosti, H., Ashikaga, E., Jaafari, N., Dearden, L., Rubin, P., Wilkinson, K. A., et al. (2012). Enhanced SUMOylation and SENP-1 protein levels following oxygen and glucose deprivation in neurones. J. Cereb. Blood Flow Metab. 32, 17-22. doi: $10.1038 /$ jcbfm.2011.146

D’Amelio, M., Cavallucci, V., Middei, S., Marchetti, C., Pacioni, S., Ferri, A., et al. (2011). Caspase-3 triggers early synaptic dysfunction in a mouse model of Alzheimer's disease. Nat. Neurosci. 14, 69-76. doi: 10.1038/nn.2709

Deacon, R. M. J., Cholerton, L. L., Talbot, K., Nair-Roberts, R. G., Sanderson, D. J., Romberg, C., et al. (2008). Age-dependent and -independent behavioral deficits in Tg2576 mice. Behav. Brain Res. 189, 126-138. doi: 10.1016/j.bbr.2007. 12.024

Di Domenico, F., Coccia, R., Butterfield, D. A., and Perluigi, M. (2011). Circulating biomarkers of protein oxidation for Alzheimer disease: expectations within limits. Biochim. Biophys. Acta 1814, 1785-1795. doi: 10.1016/j.bbapap.2011. 10.001

Dorval, V., and Fraser, P. E. (2006). Small ubiquitin-like modifier (SUMO) modification of natively unfolded proteins tau and alpha-synuclein. J. Biol. Chem. 281, 9919-9924. doi: 10.1074/jbc.M510127200

Dorval, V., and Fraser, P. E. (2007). SUMO on the road to neurodegeneration. Biochim. Biophys. Acta 1773, 694-706. doi: 10.1016/j.bbamcr.2007. 03.017
Droescher, M., Chaugule, V. K., and Pichler, A. (2013). SUMO rules: regulatory concepts and their implication in neurologic functions. Neuromolecular Med. 15, 639-660. doi: 10.1007/s12017-013-8258-6

Feligioni, M., Brambilla, E., Camassa, A., Sclip, A., Arnaboldi, A., Morelli, F., et al. (2011). Crosstalk between JNK and SUMO signaling pathways: deSUMOylation is protective against $\mathrm{H}(2) \mathrm{O}(2)$-induced cell injury. PLOS ONE 6:e28185. doi: 10.1371/journal.pone.0028185

Feligioni, M., Nishimune, A., and Henley, J. M. (2009). Protein SUMOylation modulates calcium influx and glutamate release from presynaptic terminals. Eur. J. Neurosci. 29, 1348-1356. doi: 10.1111/j.1460-9568.2009.06692.x

Feligioni, M., and Nisticò, R. (2013). SUMO: a (Oxidative) stressed protein. Neuromolecular Med. 15, 707-719. doi: 10.1007/s12017-013-8266-6

Georgopoulou, N., McLaughlin, M., McFarlane, I., and Breen, K. C. (2001). The role of post-translational modification in beta-amyloid precursor protein processing. Biochem. Soc. Symp. 23-36.

Heun, R., Schoepf, D., Potluri, R., and Natalwala, A. (2013). Alzheimer's disease and co-morbidity: increased prevalence and possible risk factors of excess mortality in a naturalistic 7-year follow-up. Eur. Psychiatry? 28, 40-48. doi: 10.1016/j.eurpsy.2011.06.001

Holman, D., Feligioni, M., and Henley, J. M. (2007). Differential redistribution of native AMPA receptor complexes following LTD induction in acute hippocampal slices. Neuropharmacology 52, 92-99. doi: 10.1016/j.neuropharm.2006.05.022

Hsiao, K., Chapman, P., Nilsen, S., Eckman, C., Harigaya, Y., Younkin, S., et al. (1996). Correlative memory deficits, Abeta elevation, and amyloid plaques in transgenic mice. Science 274, 99-102. doi: 10.1126/science.274.5284.99

Irizarry, M. C., McNamara, M., Fedorchak, K., Hsiao, K., and Hyman, B. T. (1997). APPSw transgenic mice develop age-related A beta deposits and neuropil abnormalities, but no neuronal loss in CA1. J. Neuropathol. Exp. Neurol. 56, 965-973. doi: 10.1097/00005072-199709000-00002

Izco, M., Martínez, P., Corrales, A., Fandos, N., García, S., Insua, D., et al. (2014). Changes in the brain and plasma $A \beta$ peptide levels with age and its relationship with cognitive impairment in the APPswe/PS1dE9 mouse model of Alzheimer's disease. Neuroscience 263C, 269-279. doi: 10.1016/j.neuroscience.2014. 01.003

Jaafari, N., Konopacki, F. A., Owen, T. F., Kantamneni, S., Rubin, P., Craig, T. J., et al. (2013). SUMOylation is required for glycine-induced increases in AMPA receptor surface expression (ChemLTP) in hippocampal neurons. PLoS ONE 8:e52345. doi: 10.1371/journal.pone.0052345

Jacobsen, J. S., Wu, C., Redwine, J. M., Comery, T. A., Arias, R., Bowlby, M., et al. (2006). Early-onset behavioral and synaptic deficits in a mouse model of Alzheimer's disease. Proc. Natl. Acad. Sci. U.S.A. 103, 5161-5166. doi: 10.1073/pnas.0600948103

Kawarabayashi, T., Younkin, L. H., Saido, T. C., Shoji, M., Ashe, K. H., and Younkin, S. G. (2001). Age-dependent changes in brain, CSF, and plasma amyloid (beta) protein in the Tg2576 transgenic mouse model of Alzheimer's disease. J. Neurosci. 21, 372-381.

Klingner, M., Apelt, J., Kumar, A., Sorger, D., Sabri, O., Steinbach, J., et al. (2003). Alterations in cholinergic and non-cholinergic neurotransmitter receptor densities in transgenic Tg2576 mouse brain with beta-amyloid plaque pathology. Int. J. Dev. Neurosci. 21, 357-369. doi: 10.1016/j.ijdevneu.2003.08.001

Krumova, P., Meulmeester, E., Garrido, M., Tirard, M., Hsiao, H.-H., Bossis, G., et al. (2011). Sumoylation inhibits alpha-synuclein aggregation and toxicity. J. Cell Biol. 194, 49-60. doi: 10.1083/jcb.201010117

Lee, L., Sakurai, M., Matsuzaki, S., Arancio, O., and Fraser, P. (2013). SUMO and Alzheimer's disease. Neuromolecular Med. 15, 720-736. doi: 10.1007/s12017013-8257-7

Leitao, B. B., Jones, M. C., and Brosens, J. J. (2011). The SUMO E3-ligase PIAS1 couples reactive oxygen species-dependent JNK activation to oxidative cell death. FASEB J. 25, 3416-3425. doi: 10.1096/fj.11-186346

Lesné, S., Koh, M. T., Kotilinek, L., Kayed, R., Glabe, C. G., Yang, A., et al. (2006). A specific amyloid-beta protein assembly in the brain impairs memory. Nature 440, 352-357. doi: 10.1038/nature04533

Li, Y., Wang, H., Wang, S., Quon, D., Liu, Y.-W., and Cordell, B. (2003). Positive and negative regulation of APP amyloidogenesis by sumoylation. Proc. Natl. Acad. Sci. U.S.A. 100, 259-264. doi: 10.1073/pnas.0235361100

Marcus, J. N., and Schachter, J. (2011). Targeting post-translational modifications on tau as a therapeutic strategy for Alzheimer's disease. J. Neurogenet. 25, 127-133. doi: 10.3109/01677063.2011.626471 
Markesbery, W. R. (1997). Oxidative stress hypothesis in Alzheimer's disease. Free Radic. Biol. Med. 23, 134-147. doi: 10.1016/S0891-5849(96)00629-6

Martin, S., Nishimune, A., Mellor, J. R., and Henley, J. M. (2007). SUMOylation regulates kainate-receptor-mediated synaptic transmission. Nature 447, 321-325. doi: 10.1038/nature05736

McMillan, L. E., Brown, J. T., Henley, J. M., and Cimarosti, H. (2011). Profiles of SUMO and ubiquitin conjugation in an Alzheimer's disease model. Neurosci. Lett. 502, 201-208. doi: 10.1016/j.neulet.2011.07.045

Moschos, S. J., Jukic, D. M., Athanassiou, C., Bhargava, R., Dacic, S., Wang, X., et al. (2010). Expression analysis of Ubc9, the single small ubiquitinlike modifier (SUMO) E2 conjugating enzyme, in normal and malignant tissues. Hum. Pathol. 41, 1286-1298. doi: 10.1016/j.humpath.2010. 02.007

Pittaluga, A., Feligioni, M., Longordo, F., Luccini, E., and Raiteri, M. (2006). Trafficking of presynaptic AMPA receptors mediating neurotransmitter release: neuronal selectivity and relationships with sensitivity to cyclothiazide. Neuropharmacology 50, 286-296. doi: 10.1016/j.neuropharm.2005. 09.004

Pittaluga, A., Segantini, D., Feligioni, M., and Raiteri, M. (2005). Extracellular protons differentially potentiate the responses of native AMPA receptor subtypes regulating neurotransmitter release. Br. J. Pharmacol. 144, 293-299. doi: 10.1038/sj.bjp.0705960

Sclip, A., Antoniou, X., Colombo, A., Camici, G. G., Pozzi, L., Cardinetti, D., et al. (2011). c-Jun $\mathrm{N}$-terminal kinase regulates soluble $\mathrm{A} \beta$ oligomers and cognitive impairment in AD mouse model. J. Biol. Chem. 286, 43871-43880. doi: 10.1074/jbc.M111.297515

Sclip, A., Arnaboldi, A., Colombo, I., Veglianese, P., Colombo, L., Messa, M., et al. (2013). Soluble A $\beta$ oligomer-induced synaptopathy: c-Jun N-terminal kinase's role. J. Mol. Cell Biol. 5, 277-279. doi: 10.1093/jmcb/mjt015

Steffan, J. S., Agrawal, N., Pallos, J., Rockabrand, E., Trotman, L. C., Slepko, N., et al. (2004). SUMO modification of huntingtin and huntington's disease pathology. Science 304, 100-104. doi: 10.1126/science.1092194
Tiraboschi, P., Hansen, L. A., Thal, L. J., and Corey-Bloom, J. (2004). The importance of neuritic plaques and tangles to the development and evolution of AD. Neurology 62, 1984-1989. doi: 10.1212/01.WNL.0000129697.01779.0A

Tosun, D., Schuff, N., Mathis, C. A., Jagust, W., and Weiner, M. W. (2011). Spatial patterns of brain amyloid-beta burden and atrophy rate associations in mild cognitive impairment. Brain 134, 1077-1088. doi: 10.1093/brain/ awr044

Yun, S.-M., Cho, S.-J., Song, J. C., Song, S. Y., Jo, S. A., Jo, C., et al. (2013). SUMO1 modulates A $\beta$ generation via BACE1 accumulation. Neurobiol. Aging 34, 650-662. doi: 10.1016/j.neurobiolaging.2012.08.005

Zhang, Y.-Q., and Sarge, K. D. (2008). Sumoylation of amyloid precursor protein negatively regulates Abeta aggregate levels. Biochem. Biophys. Res. Commun. 374, 673-678. doi: 10.1016/j.bbrc.2008.07.109

Conflict of Interest Statement: The authors declare that the research was conducted in the absence of any commercial or financial relationships that could be construed as a potential conflict of interest.

Received: 28 February 2014; accepted: 19 March 2014; published online: 07 April 2014. Citation: Nisticò R, Ferraina C, Marconi V, Blandini F, Negri L, Egebjerg $J$ and Feligioni M (2014) Age-related changes of protein SUMOylation balance in the A $\beta P P$ Tg2576 mouse model of Alzheimer's disease. Front. Pharmacol. 5:63. doi: 10.3389/ fphar.2014.00063

This article was submitted to Experimental Pharmacology and Drug Discovery, a section of the journal Frontiers in Pharmacology.

Copyright (C) 2014 Nisticò, Ferraina, Marconi, Blandini, Negri, Egebjerg and Feligioni. This is an open-access article distributed under the terms of the Creative Commons Attribution License (CC BY). The use, distribution or reproduction in other forums is permitted, provided the original author(s) or licensor are credited and that the original publication in this journal is cited, in accordance with accepted academic practice. No use, distribution or reproduction is permitted which does not comply with these terms. 